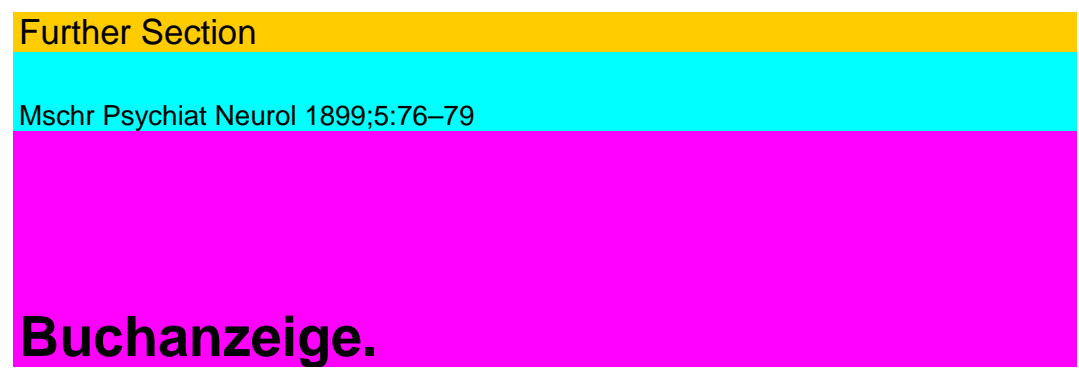

A. Pick, Beiträge zu Pathologie und pathologischen Anatomie des centralen Nervensystems mit Bemerkungen zur normalen Anatomie desselben. Mit 205 Abbildungen. Berlin 1898, S. Karger.

Das Buch enthält neben einigen schon früher in Zeitschriften veröffentlichten klinischen Studien, welche hier erweitert und durch die Ergebnisse der anatomischen Untersuchung ergänzt sind, eine grosse Zahl bisher unveröffentlichter Beobachtungen und Erörterungen.

Eine Fülle wertvoller Fälle gelangt zu klinischer Darstellung, in der Mehrzahl mit Sectionsbefund.

Einen besonderen Wert verleiht dem Buch der Reichtum der herangezogenen und discutierten Litteratur. Die erstaunliche Belesenheit des Verf. im französischen, englischen und italienischen Schrifttum gestattet ihm, die ausländischen Forschungsergebnisse in einem Umfange heranzuziehen, wie $\mathrm{e}=$ kaum in einem zweiten Werke unseres Specialgebietes geschieht. Zu zwei Dritteln handelt das Buch über Störungen der Sprache und verwandte Erkrankungen der localisierbaren psychischen Functionen.

Nachdem durch Broca, Wernicke und Lichtheim die Sprachpathologie in ihren grossen Zügen begründet und die Haupttypen der Störungen aufgestellt sind, harren noch eine Menge Detailfragen ihrer Erledigung. Die Menge der Misch- und Uebergängsfälle lässt sich keinem der grossen Typen einordnen. Feinere Nuancen der Symptome, Einzelheiten im Ablaut der Störungen fordern Aufklärung. $\mathrm{Zu}$ dieser Arbeit des feineren Ausbaus liefert Pick hier einen reichen Beitrag. Unvoreingenommen zergliedert er die Fälle, wie sie in der Klinik vorkamen, nach ihren Einzelheiten. Naturgemäss bestehen für die Deutung dieser ,unreinen" Fälle, namentlich der vielen mit allgemeiner Beeinträchtigung der Psyche, entsprechend allgemeiner Gehirnatrophie oder multiplen Herden, besondere Schwierigkeiten. Nur die Zugrundelegung einer grossen Zahl solcher Fälle giebt einigermassen Gewähr für sichere Resultate, wobei allerdings unvermeidlich auch weniger sorgfältig untersuchte Fälle mit unterlaufen, auf die etwa erst der Obductionsbefund die Aufmerksamkeit lenkte.

P. ist sich der Schwierigkeit des Unternehmens bewusst, und spricht sich daher überall mit grösster Reserve aus. Er beansprucht sicher selbst nicht, dass wir alle seine Ergehnisse für einwandfrei ansehen.

In dem einem Referat gesteckten Grenzen lässt sich natürlich nur ein unvollständiges Bild von dem Inhalt des Buches geben.

Von den Sprachstörungen hat Gelegenheit und Interesse Pick besonders auf die sensorischen geführt. Mehr als drei Viertel der sprachpathologischen Arbeiten sind der Worttaubheit resp. Blindheit gewidmet. Nicht weniger als 11 Kapitel handeln davon. 
Buchanzeige. 
Das Buch beginnt mit einem Kapitel über Störung der Identification (Asymbolie, Apraxie und Agnosie): Bei 61 jährigem Manne: Partielle optische und taktile Seelenblindheit, Seelengerucns- und -geschmackslosigkeit, massige Paraphasie bei reichem Wortschatz. Rechtsseitige Lähmungs-erscheinungen. Gesichtsfeldeinschränkung für gröbere Prüfung nach unten. Erhaltenes Formerkennen sowohl für Auge wie für Tastsinn. Dabeji gänzlich erhaltenes Sprachverständnis.

Dies macht den Fall zu einem ungewöhnlichen und dadurch ist der Kranke zugänglicher als komplet Asymbolische.

Section: Doppelseitige Hinterhauptsherde.

Rechts: hintere Gyr. fus. und ling.

Links: Cuneus und hinteres Drittel des Gyr. ling.

Mikroskopische Untersuchung wird in Aussicht gestellt.

Kapitel 2: Studien zur Lehre vorn Sprachverständnis, und Kapitel 4: Zur Lehre von der Symptomatologie doppelseitiger Schläfenlappenläsionen, sog. subcorticale sensorische Aphasie, Kapitel 6: Zur Lehre von der sub-corticalen sensorischen Aphasie, Kapitel 8: Von den partiellen Störungen des acustischen Wortcentrums und deren Beziehungen zur transcorticalen sensorischen Aphasie, und Kapitel 9: Ueber Agrammatismus als Folge cerebraler Herderkrankung, bilden eine zusammenhängende Argumentation, das Wesen und die Localisation der verschiedenen Arten von Worttaubheit betreffend. Eine Reihe selbstbeobachteter und sezierter Fälle und die Berücksichtigung fremder Befunde führen P. zu der Ansicht, dass es sich bei den Symptomenbildern der sog. corticalen, transcorticalen, subcorticalen Aphasie um verschiedene Grade der Zerstörung der beiden Schläfenlappen handele.

Die sog. transcorticale sensorische Aphasie beruhe auf partieller Läsion des linksseitigen acustischen Wortcentrums; die corticale auf totaler Läsion desselben; die sog. subcorticale senagBiSche auf partieller beiderseitiger Läsion der acustischen Centren; die corticale Taubheit auf totaler Läsion beider Hörcentren (siehe die Zusammenfassung in Kapitel 7)

Es sind also wesentlich Differenzen desUmfanges der Läsion, welche nach P. sowohl die verschiedenen Formen der $\mathrm{W}$ äroaubheit von einander, wie diese zusammen von der centralen Taubheit- unterscheiden.

P. weist auf die Analogie dieser verschiedenen staaaafljggn Formen von organisch bedingter Sprachtaubheit mit den Stadien in der RückbiHuiig von functioneller Worttaubheit hin und kommt dabei auf verschiedene Spielarten der Worttaubheit zu sprechen.

Er hatte in der Lösung eines epileptischen Anfalls nach einander beobachtet: 1. Völliges Fehlen des Verständnisses, 2. Fehlen mit automatischer Wiederholung der Frage (Echolalie;, 3. mit bewusst fragender Wiederholung.

Stadium 1 entspricht der corticalen Worttaubheit, 2. dem gewöhnlichen Bilde der transcorticalen 3 einer Abart der letzteren, für we'che P. einen Beleg beibringen kann: Das Wortverständnis war hochgradig gestört, der Wortschatz reduciert, das Nachsprechen meist fehlerlos und dabei fragendes Wiederholen der unverstandenen Worte.

Obduction: Allgemeine Atrophie mit besonders starker Beteiligung des linken Schläfenlappens und der Broca'schen Windung.

Eine weitere durch partielle Schädigung des akustischen Wortcentrums und als Phase in der Rückbildung der sensorischen Aphasie auftretende Erscheinung sieht P. in dem Aggramatismus. d. h. dem Mangel der syn-tactischen Fügung der Worte zu Sätzen.

Man sieht, der P.'sche Standpunkt gipfelt in der Zurückführung der Hauptformen der Sprachtaubheit auf quantitative Differenzen. Die subcorticale sensorische Aphasie ist der Punkt, in we'chem der Gegensatz dieser Auffassung gegen die Wernicke-Li chtheim'sche Lehre am schärfsten zum Ausdruck kommt. Gegen die Existenz einer solchen Form, als einseitig subcortical bedingter Sprachstörung richtet denn auch P. den grössten Teil seiner Argumentation, indem er den Nachweis versucht, dass es sich bei dem betr. klinischen Bilde immer um doppelseitige Erkrankung des Hörcentrums handele. 
Gerade aber dieser Teil der Beweisführung erscheint dem Ref. wenig überzeugend!).

Soweit P.'s eigenes Material in Betracht kommt, liegen derselben drei Fälle zu Grunde mit doppelseitigen Schlafenlappenaffectionen. (In dem a Falle Myska ist die rechtsseitige Schläfenlappenaffection nicht einmal erwiesen, sondern nur erschlossen: „Die makroskopisch constatierte Inselerweichung rechtfertigte den Schluss auf Mitbeteiligung des Stabkränzes zum Schläfenlappen.") Würden sie intra vitam den Symptomencomplex der subcorticalen sensorischen Aphasie geboten haben, so würden sie in der That eine gewisse Stütze für P.'s These abgeben.

"Wie steht es nun damit?

Die Kranke Myska (Kapitel 9) bietet keineswegs das Bild der subcorticalen Aphasie. Sie erweist sich vielmehr nach der Krankengeschichte als fast total taub. P. selbst führt als einzigen sicheren Beweis für erhaltenes Hörvermögen an, dass sie einmal das "Wort ,strafen" wiederholte. Wie kann man da von Worttaubheit sprechen?

Aehnlich. steht es mit dem zweiten Falle (Pelc Kapitel 4); auch hier nur minimale Hörreste, und von dem für die subcorticale sensorische Aphasie charakteristischen Complex: erhaltenem Tongehör bei fehlendem Sprachverständnis, nichts zu bemerken. Nur wenig günstiger steht es in Bezug auf das Hörvermögen mit dem älteren Fall Anton (Archiv f. Psych., 23). „Er hörte leiseres Rufen, Sprechen, klingende Glocke nicht, lautere Eufe. Klatschen, Glockenschall schwer, zuweilen erst nach öfterer Wiederholung", also auch hier ein ausserordentlich hoher Grad von Schwerhörigkeit. Eine exacte Bestimmung des Hörvermögens mit Stimmgabeln' fehlt

Es ist nun gerade P.'s Verdienst, darauf hingewiesen zu haben, 'dass ein hoher Grad von Schwerhörigkeit an sich das Sprachverständnis aufheben kann. Aber diese acustische Worttaubheit ist von der subcorticalen sensorischen Aphasie, der reinen Worttaubheit, bei der das Hörvermögen ausreichend zum Sprachverständnis ist, zu trennen. Sectionsbefunde von ersterer beweisen nichts für die letztere. Die drei P.'schen Fälle sind daher Beispiele für centrale Taubheit, resp. einen an Taubheit grenzenden Grad von Schwerhörigkeit.

Ueber Wortblindheit handeln Kapitel 3 und 7. Durch ersteres wird die Litteratur um einen jener seltenen Fälle bereichert, in denen bei einem Linkshänder rechtsseitiger Herd Wortblindheit machte. Neben der Wortblindheit : linksseitige Hemianopsie, partielle optische Aphasie und partielle Seelenblindheit, rechtsseitige Hemiplegie. Section: Rechts haselnussgrosser Erweichungsherd unter und hinter dem Gyrus angularis, in der Tiefe sich in diesen selbst erstreckend, links erbsengrosser Herd im Nucleus lentiformis. Nebenbei bemerkt P., dass der iu der Litteratur citierte B ennet'sche Fall nicht weiter unter den Belegen für die Combination linksseitiger Hemianopsie mit Wortblindheit figurieren könne, da es sich nicht um linksseitige, sondern um rechtsseitige Hemianopsie handelte. Erstere wurde nur' auf Grund eines irreführenden Referates im Neurologischen Centralblatt angenommen.

Kapitel 7 bringt einen interessanten Fall von Agraphie bei nur sehr leicht gestörtem Lesen und geringer Paraphasie. P. nimmt einen Herd am hinteren Ende der Insel an, wodurch das Schriftcentrum, ungeschädigt, die Bahnen von demselben nach dem, $>$ Schreibterritorium und vom acustischen zum motorischen Sprachcentrum zerstört sind. In dieser Deutung entspräche der Fall Wernicke's Leitungsagraphie. Kapitel 10. Zur sog. Lehre von der Leitungsaphasie (Wernicke) P. hatte bei einem Fall mit Paraphasie und Paragraphie bei nahezu intactem Sprachverständnis und partiellem Verständnis der Schrift einen Herd in der linken Insel und dem Gyrus angularis erwartet. Die Section ergab doppelseitige Erweichungen, und zwar links hintere Hälfte der Insel ,Rinde und Mark der hinteren drei Viertel des Gyrus temporalis I und XI,

J) fief. hat sich über diesen Gegenstand schon an anderer Stelle in: Psychiatr. Abhandl., herausgeg. v. Wernicke, Heft 7|8: „Ein Fall von reiner Sprachtaubheit" ausgesprochen. 
Personalien und Tages-Nachrichten. 
rechts im Sulcus praecentralis und Fuss der unteren und mittleren Stirnwindung." $\mathrm{P}$. führt nun aus, wie dieser Befund mit dem ihm anscheinend $\mathrm{wi}^{\wedge} \mathrm{B}$ strebenden klinischen Bilde zu vereinen sei: Nach der Anamnese hatte früher Spraelitaubheit, bestanden. Die weitgehende Rückbildung dieser Störung muss auf Substitution der zerstörten Schläfenpartie durch ein anderes Gehirnterritorium zurückzuführen sein.

Die Erklärung der hochgradigen Paraphasie trotz wiedergekehrten Sprachverständnisses bietet grosse Schwierigkeiten. P. giebt zu bedenken, ob dabei die Zerstörung der dritten i echten Stirnwindung eine Solle spiele. Die sinnreiche, auf diese sehr complicierten Verhältnisse gerichtete Erörterung lässt sich in der Kürze nicht wiedergeben.

P. entnimmt dem Fall den Fingerzeig bei der Diagnose: Inselaphasie darauf zu achten, ob vom Beginn ab keine "Worttaubheit vorlag.

Kapitel 13 : Drei Fälle von luiS' her cerebraler Herdaffeetion, welche eine allgemeine Gedächtnisschwäche herbeiführten. P. nimmt an, dass die den Anfällen vorangehenden diffusen Veränderungen der Gefässe und die chronische Meningitis den Boden für die Störung vorbereitet hatten.

Kapitel 14 bringt einen Beleg für getrennte Localisation des motorischen Teils des Sehens vom sensorischen. Ein Kranker mit linksseitiger Hemianopsie zeigte die Störung, dass er gesehene Gegenstände falsch localisierte, d. h. immer hinter dieselben griff. Augenmuskelstörungen wurden nicht bemerkt. Es fand sich ein doppelseitiger, den Lobus flaa talis inferior, Rinde und Mark betreffender Erweichungsherd, auf welchen P. die Störungen der Tiefenlocalisation bezieht.

Drei weitere von P. beschriebene Fälle mit Störungen der Tiefen-lokalisation boten einen zu diffusen antomischen Befund, als dass sie lokalisatorisch verwertet werden könnten: einmal allgemeine Cysticercosis des Gehirns, in den beiden anderen Fällen allgemeine GehimatrcUfgg Kap. XV berichtet über zwei Balkentumoren, welche entsprechend früheren Erfahrungen, keine charakteristischen Erscheinungen machen.

Kap. XIX: Sektionsbefund dreier Fälle mit Wiederkehr des verschwunden gewesenen Kniereflexes. Die Hinterstrangsdegeneration war in zwei Fällen nur massig ausgedehnt. Diese Befunde illustrieren so die Annahme, dass in solchen Fällen noch genug Reflex vermittelnde Fasern vorhanden seien, um beim Eintritt bestimmter Bedingungen wieder in Aktion treten zu können. TJeber die Natur dieser Bedingungen, ob es sich um Wegfall von Hemmungen oder um Reizungen handele, könne man noch nicht entscheiden.

Der letzte Teil.des Buches ist hauptsächlich pathologisch anatomischen Gegenständen gewidmet.

Kap. XVI: Partielle Erkrankung der Olivenzwischenschicht nebst Bemerkungen über die Fibrae arcuatae anterior. XVII. TJeber ein wenig beachtetes Fasersystem. XVIII. Zur Lehre von der Kommadegeneration in den Hintersträngen des Rückenmarkes. XX. Zur Lehre von den Tabes formen des Kindesalters. XXI. Beiträge zur Lehre von den Hemmungs und Missbildungen des menschl. Rückenmarkes bieten eine Fülle von thatsächlichem Material.

Liepmann (Breslau).

\section{Personalien und Tages-Nachrichten.}

Von Jahre $1899 \mathrm{ab}$ erscheint im Karg er'sehen Verlag eine Zeitschrift für Augenheilkunde, redigiert von Kuhnt-Königsberg und Michel-Würzburg. Monatlich wird ein Heft von etwa 5 Druckbogen ausgegeben. Der Preis des Jahrgangs beträgt $30 \mathrm{Mk}$. Wie die TJebersicht der angekündigten Aufsätze und Sammelreferate lehrt, werden auch die Beziehungen der Augenheilkunde zur Neurologie und Psychiatrie eingehend berücksichtigt.

Im Anschluss an das Oentralblatt für Nervenheilkunde und Psychiatrie erscheint künftig eine Zeitschrift für Elektrotherapie und medicinische 